\title{
Multiple processing-defective mutations in a mammalian histone pre-mRNA are suppressed by compensatory changes in U7 RNA both in vivo and in vitro
}

\author{
Ursula M. Bond, Therese A. Yario, and Joan A. Steitz \\ Howard Hughes Medical Institute, Department of Molecular Biophysics and Biochemistry, Yale University School of \\ Medicine, New Haven, Connecticut 06510 USA
}

\begin{abstract}
To study the role of base-pairing between the mammalian U7 snRNA and the highly variable histone downstream element (HDE) during the 3'-end maturation of mammalian histone pre-mRNAs, we mutated the HDE of the mouse H2A-614 gene and assayed processing in HeLa cells both in vivo and in vitro. Either a 9-nucleotide deletion or a block substitution of pyrimidines for 6 purines within the HDE abolished all 3'-end processing. Compensatory changes were introduced into a synthetic human U7 gene, whose transcripts assemble into Sm snRNPs in vivo. Suppression of the 6-purine substitution as well as a 3-purine substitution within the HDE was obtained in vivo by coexpressing the corresponding U7 suppressor RNAs and in vitro by using nuclear extracts prepared from HeLa cells containing U7 suppressor genes. Our results not only provide genetic evidence for base-pairing between the U7 snRNP and the HDE of mammalian histone pre-mRNAs but reveal an unexpected tolerance to drastic changes in the nature of the base-paired region.
\end{abstract}

[Key Words: Histone gene expression; U7 snRNP; RNA processing]

Received May 30, 1991; revised version accepted July 3, 1991.

At each round of cell division, the four core histone proteins are synthesized coordinately through regulation at the levels of transcription, RNA processing, and mRNA stability (Heintz et al. 1983; Lüscher et al. 1985; Lüscher and Schümperli 1987; Levine et al. 1988; Marzluff and Pandey 1988). Unlike most mRNAs, the replication-dependent histone mRNAs are not polyadenylated; instead, their $3^{\prime}$ ends are generated by endonucleolytic cleavage of longer primary transcripts. Two highly conserved sequence motifs in the histone pre-mRNAs are required for correct and efficient 3 '-end formation: a stem-loop immediately preceding the processing site and a purine-rich sequence located several nucleotides downstream of the mature $3^{\prime}$ end (Birnstiel and Schaufele 1988; Mowry and Steitz 1988).

Multiple factors have been implicated in the $3^{\prime}$-end processing of replication-dependent histone mRNAs. These include a hairpin-binding factor that is immunoprecipitable by anti-Sm antibodies despite the apparent absence of an RNA component (Mowry and Steitz 1987a; Vasserot et al. 1989), a heat labile factor whose steadystate levels are regulated during the cell cycle (Gick et al. 1987; Lüscher and Schümperli 1987), and a low-abundance small nuclear ribonucleoprotein particle (snRNP) of the Sm class called U7 (Galli et al. 1983; Strub and Birnstiel 1986; Mowry and Steitz 1987b; Soldati and Schümperli 1988).
The U7 snRNP appears to function in processing through a base-pairing interaction between the $5^{\prime}$ end of the U7 RNA and the purine-rich histone pre-mRNA downstream element (HDE) (Mowry and Steitz 1987b; Cotten et al. 1988). This has been demonstrated directly only in the sea urchin system, where 6 absolutely conserved nucleotides (GAAAGA) of the HDE are complementary to nucleotides 3-8 of sea urchin U7. Schaufele et al. (1986) showed that a double-point mutation in this region (GAATCA) of a sea urchin $\mathrm{H} 3$ gene, which abolished 3 '-end processing of the pre-mRNA, could be suppressed by introducing a compensatory double mutation into the $5^{\prime}$ end of sea urchin U7 RNA.

In higher eukaryotes, including mammals, the downstream element necessary for histone mRNA 3 '-end formation is defined much less rigidly than in the sea urchin (Taylor et al. 1986), although it is invariably purine rich (Fig. 1). Each HDE exhibits significant complementarity to mammalian U7 snRNA, involving some portion of the pyrimidine tract of U7 at nucleotides 12-18 from the $5^{\prime}$ end (see Fig. 1). However, unlike the sea urchin histone pre-mRNAs, the distance between the processing site and the region of complementarity to $U 7$ is highly variable. The idea that mammalian $\mathrm{U} 7$ interacts with the HDE through base-pairing is supported by loss of in vitro processing activity when the $5^{\prime}$ end of the human or mouse U7 RNA has been destroyed by RNase 
Figure 1. Base-pairing potential between mouse and human histone pre-mRNAs and U7 snRNA. Sequences of the purine-rich downstream element (HDE) of mouse and human histone genes are shown. Although additional histone gene sequences are known, only those genes whose expression has been characterized are shown here. The first nucleotide of the histone sequences $\left(5^{\prime} \rightarrow 3^{\prime}\right)$ is the first nucleotide downstream of the cleavage site; for mRNAs where multiple 3 '-end cleavage sites were mapped, the cleavage site closest to the base of the stem-loop is shown as the first nucleotide. (") Expressed mRNAs whose 3'-ends have not been mapped but are presumed to occur immediately downstream of the stem-loop. In the U7 sequence, nucleotides 617 ( $3^{\prime}$-AAGAUUUUCUCGACAUU-5') are shown; the $A$ at the $3^{\prime}$-end is the first base of the $\mathrm{Sm}$-binding site consensus sequence. The large, solid dots represent GC and AU base pairs between the $5^{\prime}$ end of U7 (top line) and the HDE. The smaller dots represent GU base pairs. Some nucleotides in the histone pre-mRNAs are bulged (shown as a base directly below the sequence) to accommodate maximum base-pairing with nucleotides $6-16$ of $U 7$; in some cases, alternative pairings would have been possible if bulging were also allowed in the U7 sequence. The compilation of mouse sequences was kindly provided by W. Marzluff (Florida State University).

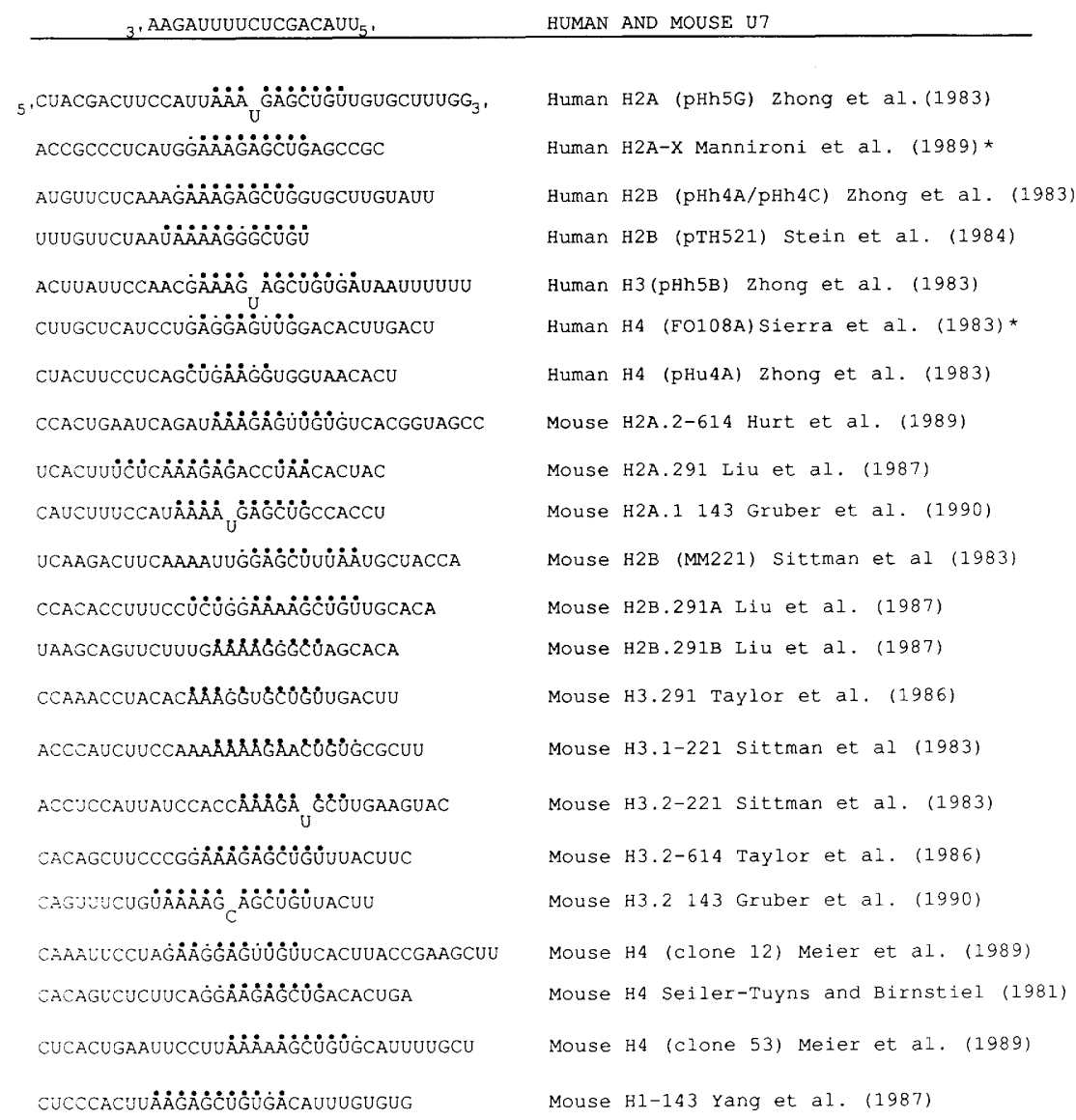

H cleavage (Mowry and Steitz 1987b; Cotten et al. 1988; Soldati and Schümperli 1988), after specific deletion of HDE sequences (Mowry et al. 1989) or upon their occlusion through hybridization with a complementary oligonucleotide (Cotten et al. 1988). Yet, no coordinate manipulation of a mammalian U7 RNA and a histone premRNA has been performed to probe the way in which the base-pairing interaction contributes to the cleavage reaction. Our experiments provide the first genetic evidence for base-pairing during the 3 '-end maturation of mammalian histone pre-mRNAs and uncover an unanticipated tolerance to drastic alteration of the conserved pairing interaction between the purine-rich region of the $\mathrm{HDE}$ and the pyrimidine stretch in U7 RNA.

\section{Results}

Mutations in the HDE of a mouse H2A gene adversely affect $3^{\prime}$-end processing

The mouse H2A-614 gene was chosen for study because it is expressed highly and processed efficiently in mouse and CHO cells (Graves et al. 1985; Levine et al. 1988; Liu et al. 1989). Its HDE contains 11 bases complementary to the mammalian U7 snRNA, including a 6-nucleotide purine stretch (Fig. 1). First, a series of deletion and substitution mutations that disrupt the base-pairing potential with $U 7$ were introduced into the purine-rich portion of the HDE (Fig. 2A,B). Their effects on pre-mRNA 3'-end processing were then examined both in vitro in a competent HeLa cell nuclear extract and in vivo by the stable introduction of each mutated gene into HeLa cells (see below).

H2A-614 substrates containing the essential hairpin loop, the processing site, and the downstream element (Fig. 2A) were incubated with various amounts of HeLa cell nuclear extract. As shown in Figure 2C (lanes 1-5), the wild-type substrate (HDEwt) is processed efficiently; $\sim 70 \%$ of the precursor is converted to product after a 60-min incubation. The appearance of two product bands is not caused by heterogeneity at the $3^{\prime}$-processed end (data not shown) but is evident in the input precursor RNA. The mutant substrate $\operatorname{HDE} \Delta 9$, which has a 9-nucleotide deletion, including the purine stretch (AAAGAG), is not detectably processed in vitro (Fig. 2C, lanes $6-10$. Note that this deletion, while disrupting the normal base-pairing with mammalian U7, in fact fortuitously generates an alternative weaker base-pairing potential (Fig. 2B); however, the distance between the site of cleavage and the complementary region in the mutant pre-mRNA is substantially reduced relative to the wild type.

A series of less drastic changes in the HDE were also 
A

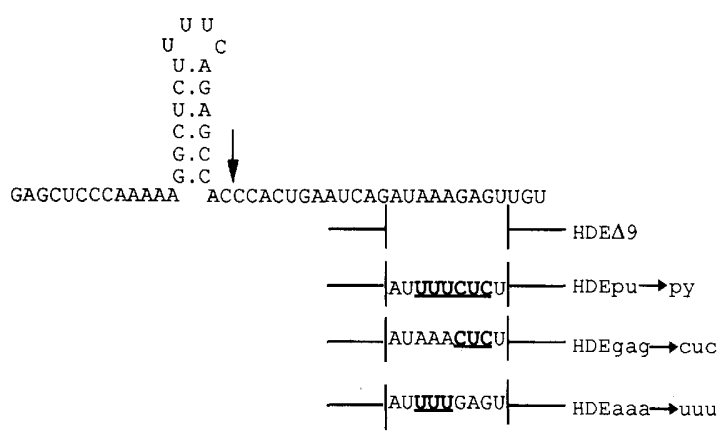

B

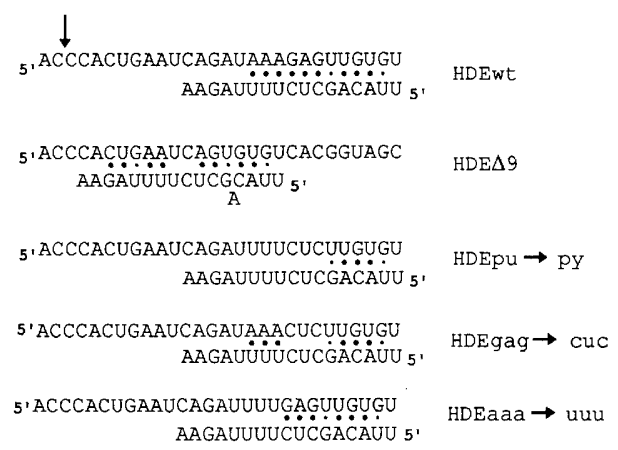

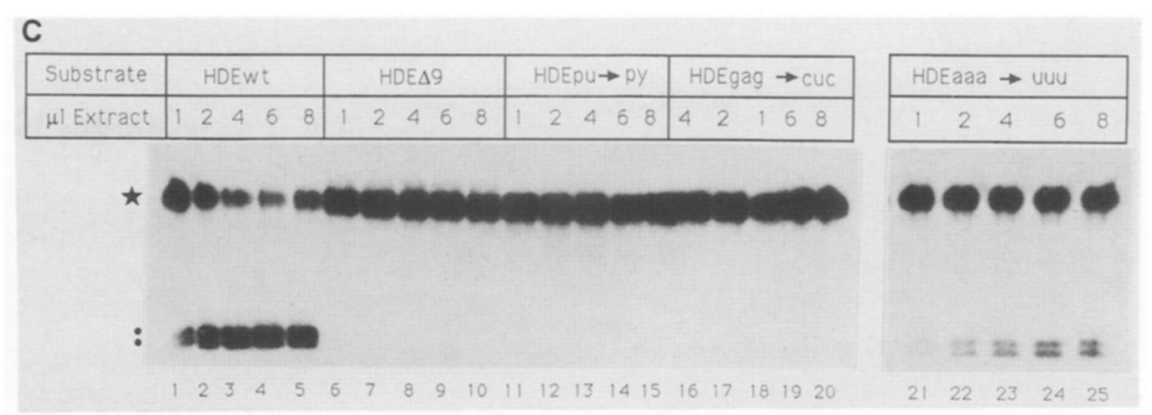

Figure 2. In vitro processing of $\mathrm{H} 2 \mathrm{~A}$ HDE mutants. (A) Sequences of the H2A-614 included in the T3 RNA polymerase runoff transcripts. The transcripts also include 14 nucleotides of vector sequence at the $5^{\prime}$ end; the total length of the transcript is 69 . The stem-loop structure upstream of the processing site (arrow) is shown. The mutations made in the HDE are indicated and the substituted bases are underlined and in boldface type. For the HDE $\Delta 9$ (which has a 9-nucleotide deletion), the runoff transcript terminated 12 nucleotides downstream of the last nucleotide shown in $A$ to generate a transcript of approximately the same size (72 nucleotides) as the other transcripts. (B) Base-pairing potential between the H2A-614 HDE mutants and wild-type human U7 snRNA. Note that the base-pairing with HDE $\Delta 9$ is not only discontinuous but requires a single-base bulge (shown as a base below the line of continuous sequence) in the U7 sequence; it is located much closer to the site of 3 '-end cleavage than most HDEs (see Fig. 1). Sequences downstream of the stem-loop of the histone mRNA and the $5^{\prime}$ end of the U7 RNA (nucleotides 6-17) are shown. An arrow marks the position of the $3^{\prime}$ cleavage site. GC and UA base pairs are depicted by large dots; GU base pairs are indicated by smaller dots. $(C)$ Products of in vitro processing of the histone mutant substrates after $60 \mathrm{~min}$ at $30^{\circ} \mathrm{C}$ in a HeLa cell nuclear extract. The RNA substrates and the amount of extract added to each 10- $\mu$ l reaction are shown at top. (Lanes 1-5) HDEwt RNA; (lanes 6-10) HDE $\Delta 9$ RNA; (lanes 11-15) HDEpu $\rightarrow$ py RNA; (lanes 16-20) HDEgag $\rightarrow$ cuc RNA; (lanes 21-25) HDEaaa $\rightarrow$ uuu RNA. The star indicates the added precursor RNA ( $\sim 70$ nucleotides); the dots indicate the products of the reaction $(\sim 45$ nucleotides). The precursors and products migrate at $\sim 78$ and 51 nucleotides, respectively, relative to pBR322 MspI molecular weight markers. The amounts of product generated were quantitated on a Phosphorimager (Molecular Dynamics). Note that lanes 16 and 18 were switched during loading of the gel; they are correct as labeled. Similar results were obtained in at least eight independent experiments.

made (Fig. 2A). Substitution of the 6-nucleotide purine stretch (AAAGAG) with its complement UUUCUC (HDEpu $\rightarrow$ py), which maintains the same distance between the cleavage site and the remainder of the potentially base-paired region, resulted in a lack of detectable processing, as did a substitution of the GAG with its complement (HDEgag $\rightarrow$ cuc) (Fig. 2, lanes 11-15 and 16-20, respectively). On the other hand, a substitution of the AAA with its complement (HDEaaa $\rightarrow$ uuu) only decreased in vitro processing to $\sim 20 \%$ (Fig. $2 \mathrm{C}$, lanes $21-$ 25).

\section{A synthetic U7 gene flanked by U1 expression signals is active in HeLa cells}

We then wanted to test whether the mutations in the HDE would have a similar effect on $3^{\prime}$-end processing in vivo and, if so, could compensatory mutations in the region of the U7 RNA that base-pairs with the HDE restore processing. As a human U7 gene had not yet been isolated, it was necessary to construct an active U7 gene into which compensatory suppressor mutations could be introduced. An oligonucleotide containing residues 3-63 of the human U7 sequence (Mowry and Steitz 1987b) was cloned downstream of a U1 gene promoter (Manser and Gesteland 1981; Hernandez 1985; kindly provided by R. Ach, Yale University). To enable us to distinguish between the endogenous U7 RNA and the product of the transfected gene, we altered nucleotides that form part of the stem of the single stem-loop structure, because similar base changes in sea urchin U7 had been shown previously not to affect its biological activity (Gilmartin et al. 1988). Because the identities of the first two nucleotides of the human U7 RNA are not known, the first 
two nucleotides of the human U1 gene (AT) were substituted. Downstream of the U7 sequences we incorporated the sequences necessary for $\mathrm{Ul} 3^{\prime}$-end formation to ensure that the $3^{\prime}$ end of the U7 RNA would be processed correctly (Hernandez 1985). The complete sequence of the inserted DNA is shown in Materials and methods (see Fig. 7A, below); Figure 3A compares the sequences of endogenous human $\mathrm{U} 7$, murine $\mathrm{U} 7$, and the synthetic U7 (U7syn) RNAs.

Suppressor mutations (Fig. 3A) were then introduced into the synthetic $\mathrm{U} 7$ gene, followed by transfection into HeLa cells with (or without) the matching H2A HDE mutant histone gene (see below). Analyses of U7 and histone RNA expression were performed by Tl RNase protection (a schematic representation of all antisense probes is shown in Materials and methods; see Fig. 8, below).

In cell lines stably expressing the various synthetic $U 7$ genes, a cluster of four to five protected RNA bands of $\sim 72,67,63$, and 62 nucleotides appear (Fig. 3B, lanes $3-5,8-11,14-15)$ compared to lines that received only the neomycin gene (lanes $2,7,13$ ). The expected size of the RNase T1 protected fragment is 63 nucleotides for all transfected $U 7$ genes (bands indicated by $\leftarrow$. The expression levels vary from cell line to cell line (Fig. 3B, cf. lanes 3 and 4 ) and range between approximately one-fifth and fivefold of the level of the endogenous U7 (major protected fragments are designated by $\mathrm{Ol}_{\text {; }}$ a severalfold variation in relative levels is observed even in duplicate analyses of the same cell line. Note that because the probes reflect base changes introduced into the U7syn gene, the sizes of protected fragments generated by the endogenous U7 vary (see Fig. 3A).

We then assessed the ability of U7syn and mutant
RNAs to assemble into RNPs by immunoprecipitating nuclear extracts prepared from the various cell lines with anti-Sm antibodies. Protection analyses, again with antisense U7 probes, showed that all but the largest transcript (72 nucleotides) resulting from the transfected U7 genes (bands indicated by $\rightarrow$ ) are immunoprecipitated (Fig. 3C). The degree of immunoprecipitation varied from extract to extract, but in each case approximately the same percentage of endogenous and transfected U7 was immunoprecipitated [cf. $\rightarrow$ with $\bigcirc$ bands in $\mathrm{P}$ (precipitate) and S (supernatant) lanes].

\section{Suppressor mutations in U7 RNA can rescue $3^{\prime}$-end processing of mutant H2A mRNAs in vivo}

Cell lines expressing the wild-type H2A-614 or mutated histone genes alone or together with the appropriate $\mathrm{U} 7$ suppressor gene were obtained. RNase protection analyses of three independent cell lines expressing the wildtype histone gene alone indicated that the pre-mRNA is processed correctly in HeLa cells (Fig. 4A, HDEwt; cf. lanes 4-6 and lane 3, neomycin only). The cluster of bands at $\sim 155$ nucleotides $(\rightarrow)$ correlates well with the size expected (160 nucleotides) for the protected fragment, with some nibbling of the termini. Only a trace $(1-5 \%)$ of readthrough transcripts is apparent (indicated by ${ }^{*}$.

In contrast, analyses of three of five independent cell lines expressing the 9-bp deletion of the H2A HDE (Fig. $4 \mathrm{~A}, \mathrm{HDE} \Delta 9$; cf. lanes 9-11 and lane 8, neomycin only) showed a fragment of $\sim 230$ nucleotides $\left({ }^{*}\right)$. This is close to the length expected for unprocessed readthrough transcripts (240 nucleotides) that are protected by histone antisense sequences in the probe. The relative usage of

Figure 3. (A) Sequences of the mouse, human, and synthetic U7 RNA. Underlined letters represent changes between the endogenous human $U 7$ and the transfected U7syn or the mouse U7. The bases below the mouse U7 sequence indicate differences between the two published mouse sequences (Cotten et al. 1988; Soldati and Schümperli 1988). The $Y$ at the 3 ' end of the mouse sequence indicates a pyrimidine. The base-pairing potential of the $3^{\prime}$-stem regions is indicated by the arrows. The mutations introduced into the U7syn gene are shown. Below the RNA sequences are indicated the positions and sizes of RNase T1 protection products expected to arise when the various antisense U7 mutant probes pair with the endogenous U7 RNA: The arrows above the lines represent primary cleavages; the arrows below indicate secondary cleavages of the primary protected fragments. Note that the complementarity of the fully protected T1 RNase product of the transfected genes extends 1 nucleotide beyond the $5^{\prime}$ end and 3 nucleotides beyond the 3 ' end of the U7 RNA (see Fig. 7A). (B) RNase protection analyses of cell lines transfected with the U7 suppressor genes. The antisense probes (all 130 nucleotides but migrate at 140-150 nucleotides relative to DNA markers) used are shown at top. (Lane 1) Input antisense $\mathrm{U} 7 \mathrm{cuc} \rightarrow$ gag probe; (lanes 2,7,13) RNA from cell lines expressing the neomycin gene; (lanes 3,4) RNA from two independent cell lines expressing the U7py $\rightarrow$ pu gene; (lane 5) RNA from a cell line expressing the U7cuc $\rightarrow$ gag gene; (lane 6) input U7cuc $\rightarrow$ gcg probe; (lanes 8-11) RNA from four independent cell lines expressing the U7cuc $\rightarrow$ gcg gene (the cell line in lane 9 coexpresses the H2A HDEgag $\rightarrow$ cuc gene); (lane 12) input U7uuu $\rightarrow$ aaa probe; (lane 14) RNA from a cell line expressing the U7uuu $\rightarrow$ aaa gene (this cell line also expresses the HDEaaa $\rightarrow$ uuu gene); (lane 15) RNA from a cell line expressing the U7syn gene. The protected fragments corresponding to the transfected $U 7$ genes are marked by arrows; the fragment at $\sim 50$ nucleotides protected by the U7uuu $\rightarrow$ aaa probe (lanes 14 and 15) was not always observed and presumably results from a secondary cleavage of one of the longer protected fragments. The bands corresponding to the endogenous U7 are indicated by $\bigcirc$. pBR322 MspI molecular weight markers and their sizes are shown. The same protection products were obtained in three independent experiments. (C) Anti-Sm precipitation of transfected U7 snRNAs. Nuclear extracts were prepared from cell lines expressing U7syn or its mutants and immunoprecipitated with anti-Sm antibodies as described in Materials and methods. RNAs extracted from the supernatants (S) and pellets (P) were analyzed by T1 RNase protection by using the U7 antisense probes (all 130 nucleotides) shown at top. The cell line analyzed is also indicated above each lane. ( $\rightarrow$ ) Protected fragments resulting from the transfected genes; (O) protected fragments resulting from the endogenous U7 RNA. Lane P (with asterisk below) is the pellet from an immunoprecipitation with nonimmune serum. The sizes of the molecular weight markers (pBR322 MspI) are shown. The bands at $\sim 115$ nucleotides present in the U7syn S lane presumably are degradation products of the undigested probe. 


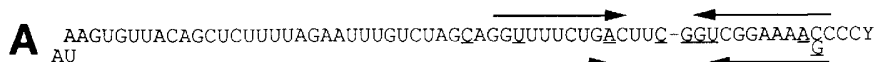
AN NGUGUUACAGCUCUUUUAGAaUUUGUCUAGUaGGCUUUCUGGCUUUUCACCGGAAAGCCCCU AUGUGUUACAGCUCUUUUAGAAUUUGUCUAGUAGGGAAUUCGGCUUUUCACCGAAUCCCCCU GAG

GCG

$A A A$

GAGAAA
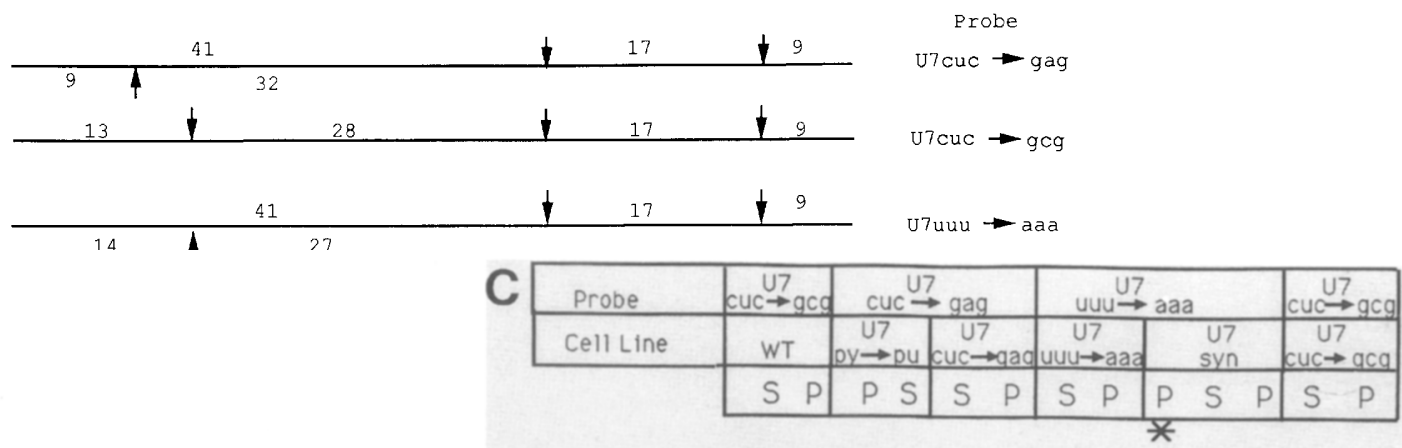

B

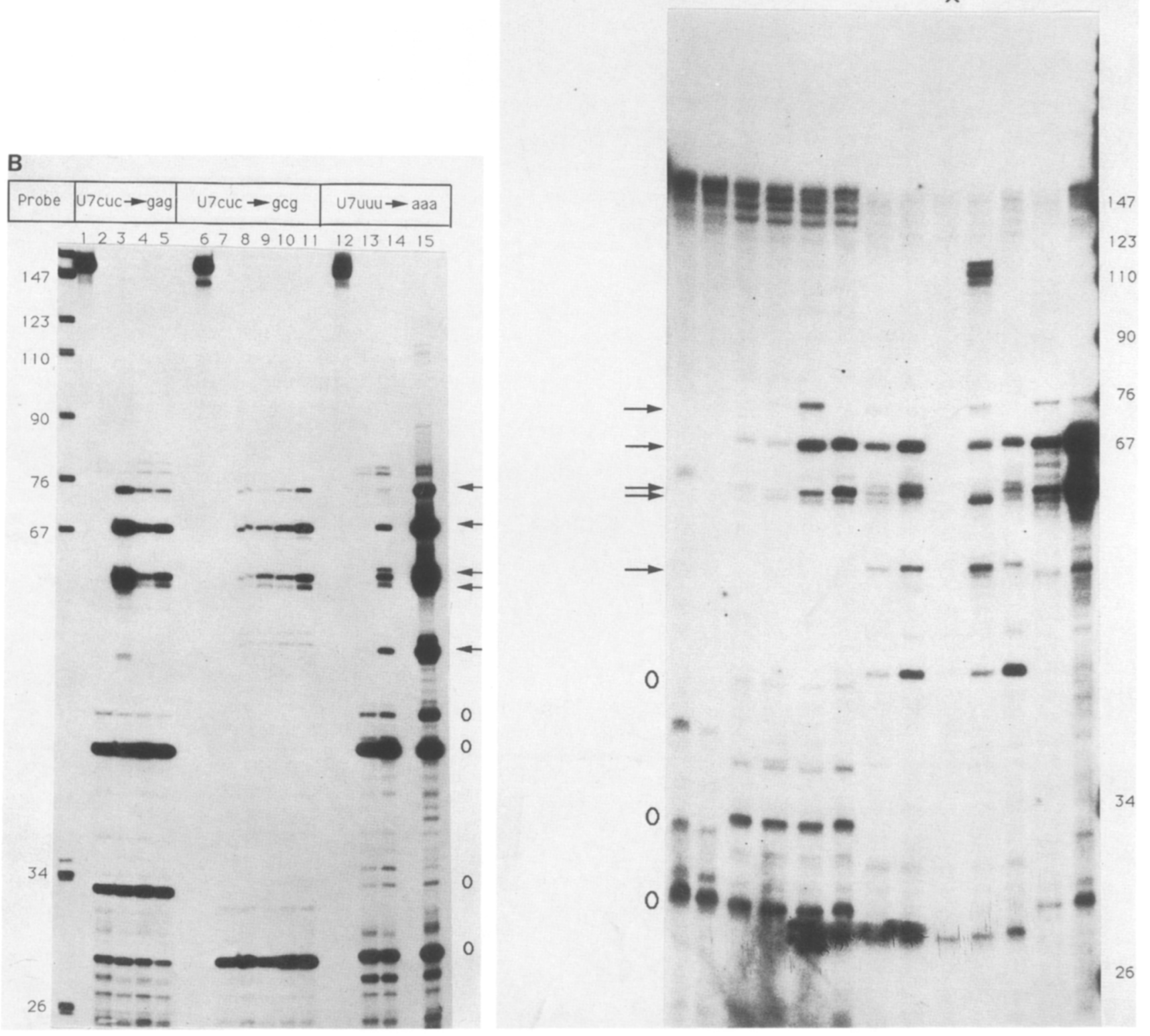

Mouse U7

Human U?

U7syn

$\mathrm{U} 7 \mathrm{cuc} \rightarrow \mathrm{gag}$

$\mathrm{U} 7 \mathrm{cuc} \rightarrow \mathrm{gcg}$

$\mathrm{U} 7 \mathrm{uuu} \rightarrow \mathrm{aaa}$

$\mathrm{v} 7 \mathrm{py} \rightarrow \mathrm{pu}$

Probe

$\mathrm{U} 7 \mathrm{cuc} \rightarrow \mathrm{gag}$

$\mathrm{U} 7 \mathrm{cuc} \rightarrow \mathrm{gcg}$

บ7uuu $\rightarrow$ aaa

Figure 3. (See facing page for legend.) 
A

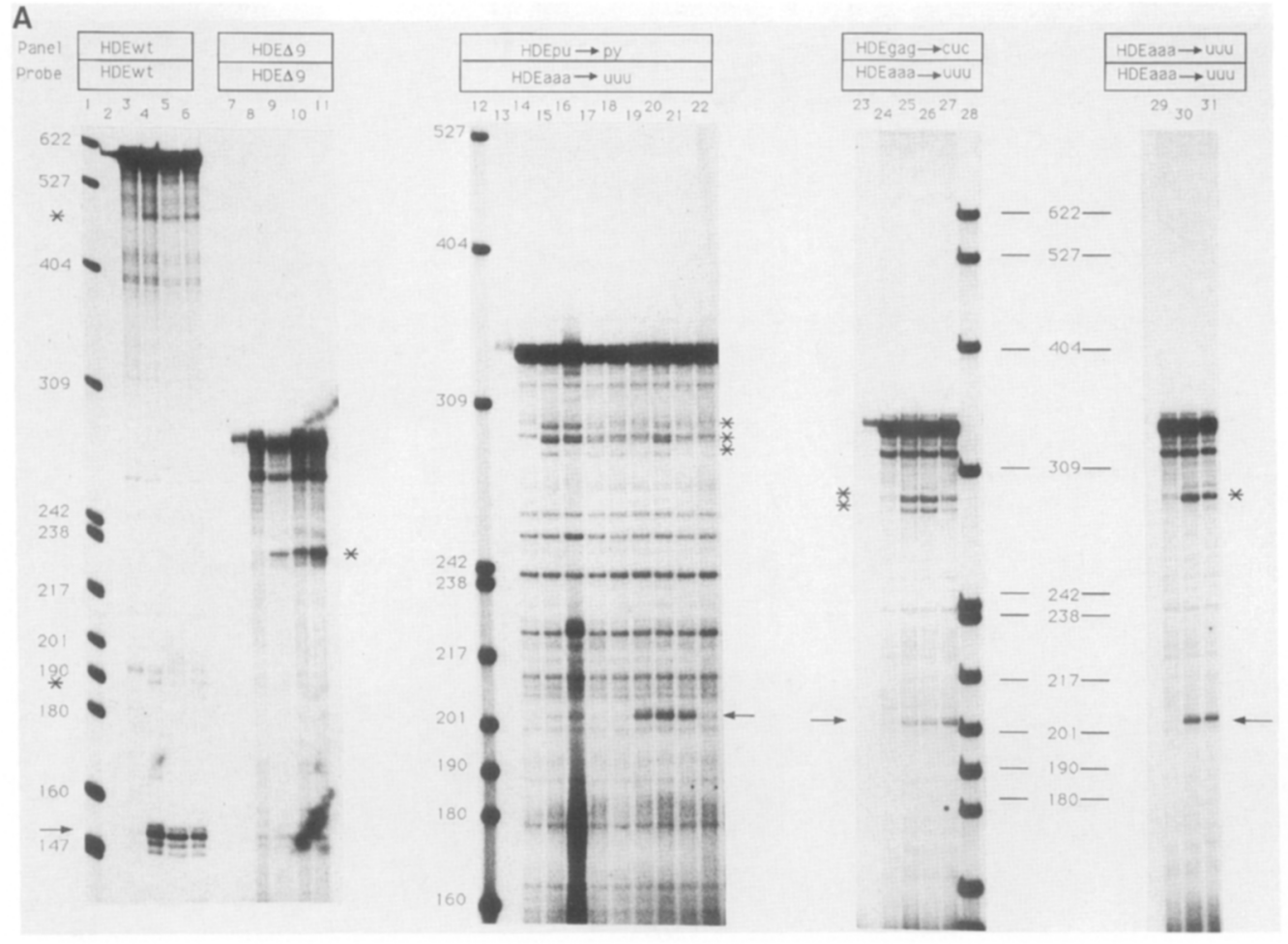

B

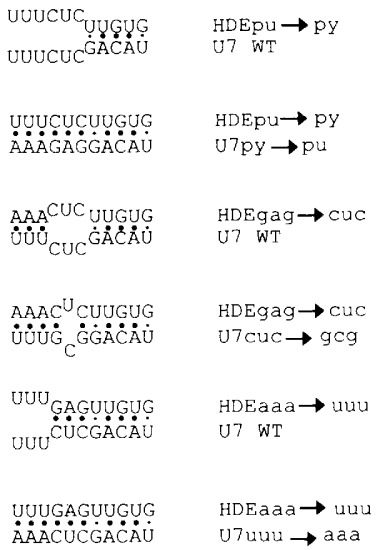

Figure 4. RNase protection analysis of the expression of $\mathrm{H} 2 \mathrm{~A}$ mutants in vivo in the presence and absence of U7 suppressor genes. $(A)$ The antisense probes used are indicated at top. $\rightarrow \mid$ The positions of the protected fragments expected for correct processing; $\left({ }^{*}\right)$ readthrough transcripts. Panel HDEwt: (Lane 1) pBR322 MspI markers; (lane 2) input antisense HDEwt probe (598 nucleotides); (lane 3) RNA from a cell line transfected with the neomycin gene only; (lanes 4-6) RNA from three independent cell lines transfected with the HDEwt gene. The same results were obtained in three other independent experiments. Panel HDE $\Delta$ : (Lane 7) Input antisense HDE $\Delta 9$ probe (296 nucleotides); (lane 8 ) RNA from a cell line transfected with the neomycin gene only; (lanes 9-11) RNA from three independent cell lines transfected with the HDE $\Delta 9$ gene (note that although a faint band at 155 nucleotides appears in lanes 9-11, it is no darker than in lane 8 ; in six independent RNase protection experiments with these cell lines no enhancement of the bands corresponding to correctly processed RNA relative to that in cell lines expressing only the neomycin gene was ever observed). Panel HDEpu $\rightarrow$ py: (Lane 12) pBR322 MspI DNA marker; (lane 13) input antisense HDEaaa $\rightarrow$ uuu probe (342 nucleotides) la 3-base bulge (CUC) between the RNA and this antisense probe exists; however, because no G residues are present in the bulge in the antisense probe, no additional RNase T1 products are expected and none are observed); (lane 14) RNA from cells transfected with the neomycin gene only; (lanes 15-17) RNA from three cell lines transfected with the HDEpu $\rightarrow$ py gene and the neomycin gene (although a small amount of correctly processed product $(\sim 210$ nucleotides $)$ is apparent in lane 15 , this was not observed in three other independent experiments.); (lane 18) RNA from the same cell line as that in lane 17, which was retransfected with the hygromycin gene; (lanes 19-22) RNA from the same cell line as that in lane 17, which was retransfected with the U7py $\rightarrow$ pu gene and the hygromycin gene. Panel HDEgag $\rightarrow$ cuc: (Lane 23) Input antisense HDEaaa $\rightarrow$ uuu probe (342 nucleotides) [a 6-base bulge (AAACUC) between the RNA and this antisense probe exists; however, as no $\mathrm{G}$ residues are present in the bulge in the antisense probe no additional RNase $\mathrm{Tl}$ products are expected and none are observed); (lane 24) RNA from a cell line expressing only the neomycin gene; (lanes 25,26) RNA from two independent cell lines expressing the HDEgag $\rightarrow$ cuc gene; (lane 27) RNA from a cell line expressing both the H2A HDEgag $\rightarrow$ cuc and the U7cuc $\rightarrow$ gcg suppressor gene; (lane 28) pBR322 MspI marker. The same results were obtained in five independent RNase protection experiments with RNA from these cell lines. Panel HDEaaa $\rightarrow$ uuu: The input probe (not shown) is the same as for panel HDEgag $\rightarrow$ cuc; (lane 29) RNA from a cell line expressing the neomycin gene only; (lane 30) RNA from a cell line expressing the HDEaaa $\rightarrow$ uuu gene only; (lane 31) RNA from a cell line coexpressing the H2A HDEaaa $\rightarrow$ uuu and the U7uuu $\rightarrow$ aaa suppressor gene. Similar results were obtained in six independent RNase protection experiments. The amount of correctly processed RNA in the HDEgag $\rightarrow$ cuc and the HDEaaa $\rightarrow$ uuu panels was quantitated by a Phosphorimager (Molecular Dynamics). $(B)$ Basepairing of HDEpu $\rightarrow$ py, HDEgag $\rightarrow$ cuc, and HDEaaa $\rightarrow$ uuu RNAs with U7 wild-type and suppressor RNAs. Large solid dots represent AU and CG base-pairing; smaller dots indicate GU base pairs.

the correct processing site (correctly processed/total protected RNA) for this and the other mutants discussed below correlates well with the efficiency of processing of the histone mutants in vitro (Table 1, cf. in vivo ${ }^{-}$with in vitro $\left.{ }^{-}\right)$.

RNase protection analyses of six independent cell lines containing the HDEpu $\rightarrow$ py mutant (three of which are shown in Fig. 4A, (HDEpu $\rightarrow$ py, lanes 15-17) likewise showed accumulation of readthrough transcripts. A cluster of bands at $\sim 295$ nucleotides $(*)$, corresponding to the length expected for full protection of the probe by complementary histone RNA sequences, 
Table 1. Processing of mutant histone pre-mRNAs

\begin{tabular}{|c|c|c|c|c|c|}
\hline \multirow[b]{2}{*}{ Histone mutant } & \multirow[b]{2}{*}{ U7 suppressor } & \multicolumn{2}{|c|}{ In vivo } & \multicolumn{2}{|c|}{ In vitro } \\
\hline & & $\sup ^{-}$ & $\sup ^{+}$ & $\sup ^{-}$ & $\sup ^{+}$ \\
\hline HDEwt & & ++++ & NA & +++ & NA \\
\hline $\mathrm{HDE} \Delta 9$ & & - & ND & - & ND \\
\hline HDEpu $\rightarrow$ py & $\mathrm{U} 7 \mathrm{py} \rightarrow \mathrm{pu}$ &,-+ & ++++ & - & ++ \\
\hline \multirow[t]{2}{*}{ HDEgag $\rightarrow$ cuc } & U7cuc $\rightarrow$ gag & ++ & ND & - & + \\
\hline & $\mathrm{U} 7 \mathrm{cuc} \rightarrow \mathrm{gcg}$ & ++ & +++ & - & + \\
\hline HDEaaa $\rightarrow$ uuu & U7uuu $\rightarrow$ aaa & +++ & +++ & +++ & +++ \\
\hline
\end{tabular}

Abbreviations: (sup) Suppressor; (NA) not applicable; (ND) not determined.

sup $^{-}$(suppressor) indicates processing in an extract containing only the endogenous U7 in vitro or HDE mutant genes transfected alone in vivo. sup ${ }^{+}$indicates processing in extracts prepared from cell lines expressing the transfected U7 genes in vitro or HDE mutant cotransfected with a U7 suppressor gene in vivo. $(++++180-100 \%$ conversion of precursor to product in vitro or correctly processed mRNA relative to the sum of correctly processed mRNA and readthrough transcripts in vivo; $1+++\mid 30-50 \% ; 1++1$ $10-20 \% ;|+| 1-5 \% ;(-\mid$ not detectable $\mid<0.5 \%)$.

and no more than $5 \%$ (and, in most cases, undetectable levels) of correctly processed RNAs (expected fragment size 210) are observed (Fig. 4A, HDEpu $\rightarrow$ py; cf. lanes 15-17 and lane 14, neomycin gene only).

Initially, we did not obtain a cell line expressing both the HDEpu $\rightarrow$ py gene and its suppressor U7py $\rightarrow$ pu (although we obtained both singly). We therefore retransfected a G-418-resistant cell line that expressed the H2A $\mathrm{HDEpu} \rightarrow$ py gene (cell line shown in Fig. 4A, lane 17) with the U7py $\rightarrow$ pu gene and a hygromycin B phosphotransferase gene (kindly provided by Dr. J. Sedivy, Yale Universityl and selected hygromycin-resistant clones. We obtained seven independent cell lines that expressed the U7py $\rightarrow$ pu gene at levels comparable to that observed previously in a cell line expressing the $\mathrm{U} 7 \mathrm{py} \rightarrow$ pu gene alone. When the HDEpu $\rightarrow$ py RNA in the presence of the U7py $\rightarrow$ pu gene was analyzed, we found that $80-90 \%$ of the transcript was processed correctly in three cell lines (HDEpu $\rightarrow$ py; Fig. 4A, cf. lanes 19-21 and lane 18, hygromycin gene alone) and 20-50\% in four other cell lines (HDEpu $\rightarrow$ py; Fig. 4A, lane 22 and data not shown).

We obtained one cell line expressing both the histone HDEgag $\rightarrow$ cuc mutant and a U7 suppressor gene (U7cuc $\rightarrow \mathrm{gcg}$ ) and two expressing this histone mutant alone. This particular $\mathrm{U} 7$ mutation is predicted to restore base-pairing with the H2A HDE except for a 1-bp mismatch at nucleotide 13 of the U7 RNA (Fig. 4B). In the absence of the U7 suppressor, only $\sim 20 \%$ of the premRNA from the transfected H2A HDEgag $\rightarrow$ cuc gene is processed at the correct site $(\rightarrow)$, with the remainder appearing as readthrough transcript $\left({ }^{*}\right)$ (HDEgag $\rightarrow$ cuc; Fig. 4A, lanes 25, 26). In the presence of the U7cuc $\rightarrow$ gcg suppressor, the yield of correct $3^{\prime}$-end processing increases to $\sim 60 \%$ (HDEgag $\rightarrow$ cuc; Fig. 4A, lane 27). Thus, the U7cuc $\rightarrow$ gcg suppressor mutation increases the efficiency of correct processing about threefold in vivo.

We also obtained a single cell line expressing both the H2A HDEaaa $\rightarrow$ uuu mutant and the corresponding $\mathrm{U} 7 \mathrm{uuu} \rightarrow$ aaa suppressor gene and one expressing this histone mutant alone. In the absence of the U7 suppressor gene, correct $3^{\prime}$-end processing of the HDEaaa $\rightarrow$ uuu mutant pre-mRNA is reduced (to $\sim 30-50 \%$ ) but not abolished (HDEaaa $\rightarrow$ uuu; Fig. 4A, lane 30). The presence of the U7uuu $\rightarrow$ aaa gene increases processing at the correct site only slightly $(1.5$-fold $)$ in the experiment shown [cf. lanes 30 (HDEaaa $\rightarrow$ uuu alone) and 31 $($ HDEaaa $\rightarrow$ uuu plus U7uuu $\rightarrow$ aaa)]; however, in two out of six experiments, no significant increase in processing was observed (data not shown).

Suppression can be reproduced in vitro with mutant histone substrates and extracts from cells expressing U7 suppressor genes

To assess the specificity of suppression, we also investigated processing of the mutant $\mathrm{H} 2 \mathrm{~A}$ histone pre-mRNAs in nuclear extracts prepared from HeLa cell lines expressing the various U7 suppressor genes (Fig. 5). As shown previously in Figure 2C (lanes 11-15), an extract containing only the endogenous U7 RNA does not process the HDEpu $\rightarrow$ py mutant pre-mRNA to any significant extent (Fig. 5A, lanes 1-4). Even after $6 \mathrm{hr}$ of incubation under limiting substrate concentration, no correctly sized products are evident. We deduce that the several bands generated in this extract arise from nonspecific degradation, as they appear even after heating the extract (data not shown, but see Fig. 5B). In contrast, the same HDEpu $\rightarrow$ py mutant substrate is processed correctly in an extract containing the U7py $\rightarrow$ pu suppressor (Fig. 5A, lanes 5-8). The low efficiency $(\sim 10 \%$ after $6 \mathrm{hr}$ of incubation/ cannot be ascribed to an inactive extract because the same extract processes the wild-type $\mathrm{H} 2 \mathrm{~A}$ (HDEwt) substrate efficiently, with $80 \%$ of the precursor being converted to product (Fig. 5A, lanes 13-16). (A comparison of the initial rates of the reaction, when conversion of precursor to product is linear, indicates that the mutant $\mathrm{HDEpu} \rightarrow$ py is processed at a rate $3 \%$ that of the HDEwt.) An extract expressing a different U7 suppressor gene (U7cuc $\rightarrow \mathrm{gcg}$ ) does not process the $\mathrm{H} 2 \mathrm{~A}$ 

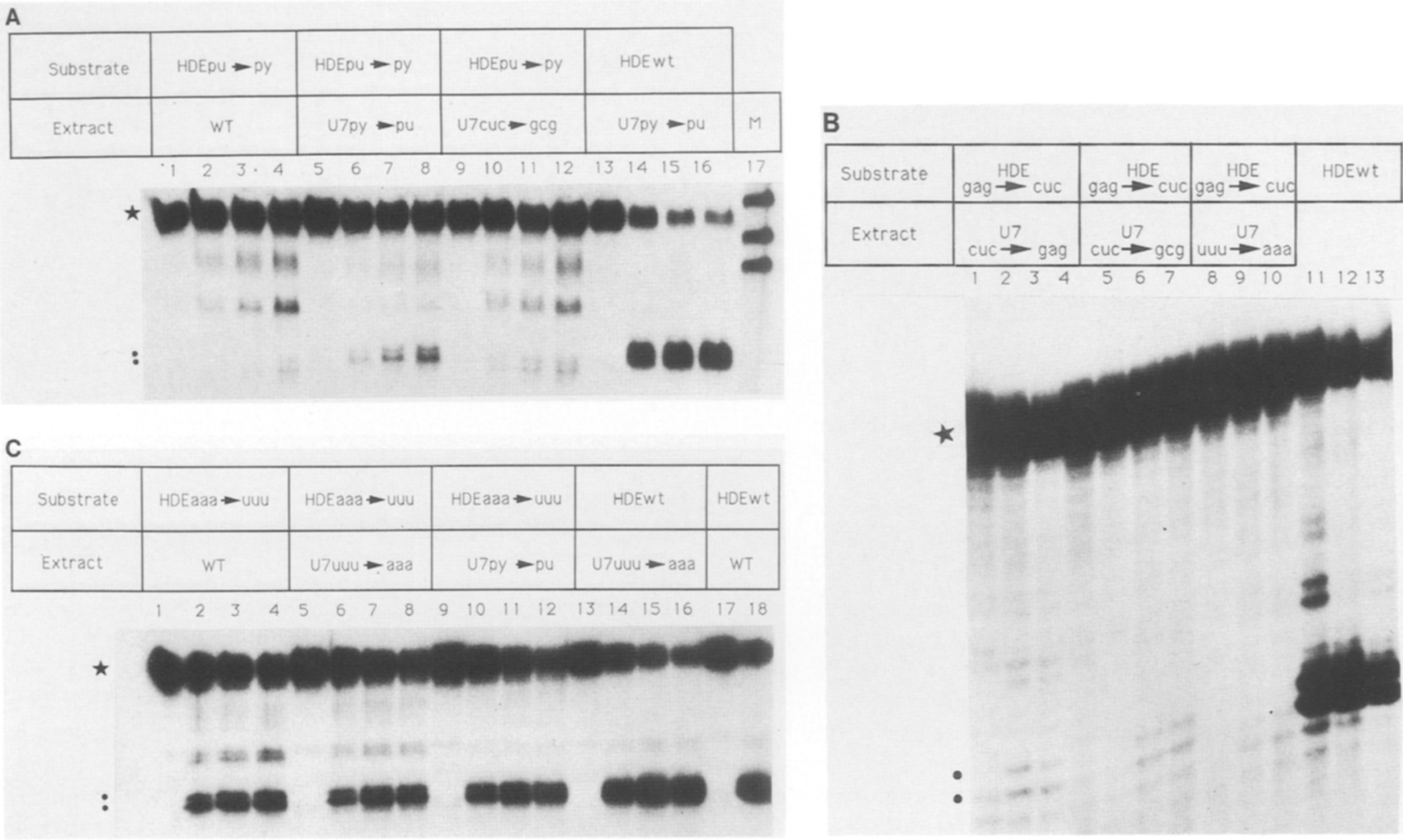

Figure 5. Time course of in vitro processing of HDE mutant substrates in extracts from cell lines expressing U7 suppressor genes. The substrates (all T3 RNA polymerase runoff transcripts) and the extract used are shown at top. The extracts were incubated for the following times at $30^{\circ} \mathrm{C}$. $|A|$ (Lanes $1,5,9,13 \mid 0 \mathrm{~min}$; (lanes 2,6,10,14) $1 \mathrm{hr}$; (lanes 3,7,11,15) $3 \mathrm{hr}$; (lanes 4,8,12,16) $6 \mathrm{hr}$; (lane 17) pBR322 MspI markers, 90, 76, and 67 nucleotides from top to bottom. (B) (Lanes 1,5,8) 0 min; (lanes 2,6,9) $2.5 \mathrm{hr}$; (lane 4) extract was pretreated at $50^{\circ} \mathrm{C}$ for $15 \mathrm{~min}$ prior to incubation with substrate for $2.5 \mathrm{hr}$; (lanes $3,7,10$ ) $5 \mathrm{hr}$; (lane 11) $2.5 \mathrm{hr}$ in a U7 wild-type extract; (lane 12) $2.5 \mathrm{hr}$ in U7cuc $\rightarrow$ gag extract; (lane 13) $2.5 \mathrm{hr}$ in U7cuc $\rightarrow$ gcg extract. (C) Lanes $1-16$ are as described for $A$; (lane 17), 0 min; (lane 18) $1 \mathrm{hr} .\left(^{*}\right)$ The input precursor RNA $(\sim 70$ nucleotides); the dots show the doublet of processed RNA (45 nucleotides). Both the precursor and product migrate slightly slower than expected relative to DNA pBR322 MspI markers. The gels were quantitated with a Phosphorimager (Molecular Dynamics). Extracts varied by no more than $20 \%$ in their efficiency of processing of the wild-type substrate. The same results were obtained in at least two independent experiments.

HDEpu $\rightarrow$ py pre-mRNA to any detectable extent (Fig. $5 \mathrm{~A}$, lanes 9-12).

Compared to an extract containing only the endogenous U7 (Fig. 2C, lanes 16-20), a slight increase of correctly processed HDEgag $\rightarrow$ cuc substrate (to $\sim 1 \%$ ) is detected after $6 \mathrm{hr}$ in extracts prepared from cells expressing the U7cuc $\rightarrow$ gag (Fig. 5B, lanes 1-3) or the U7cuc $\rightarrow$ gcg (lanes 5-7) suppressor gene. (The rates of initial processing are $\sim 0.3 \%$ and $0.2 \%$, respectively, compared to that of the HDEwt substrate.) This low level of processing is abolished by pretreatment of the $\mathrm{U} 7 \mathrm{cuc} \rightarrow$ gag suppressor extract at $50^{\circ} \mathrm{C}$ for $15 \mathrm{~min}$ (lane 4), a treatment that abolishes histone 3 '-end processing (Gick et al. 1987). In contrast, in an extract prepared from cells expressing a noncomplementary U7 suppressor (U7uuu $\rightarrow$ aaa), no product from the HDEgag $\rightarrow$ cuc RNA can be observed (lanes 8-10). Extracts containing the U7cuc $\rightarrow$ gag and $\mathrm{U} 7 \mathrm{cuc} \rightarrow \mathrm{gcg}$ suppressors processed the HDEwt substrate as efficiently as a U7 wildtype extract (Fig. 5B, cf. lanes 12 and 13 and lane 11).
Processing of the H2A HDEaaa $\rightarrow$ uuu mutant RNA, which is $-20-30 \%$ the efficiency of the wild-type $\mathrm{H} 2 \mathrm{~A}$ substrate in an extract containing only endogenous U7 (Fig. 5C, cf. lanes 1-4 and 17-18; also see Fig. 2C), is not increased in an extract containing the U7uuu $\rightarrow$ aaa suppressor RNA (lanes 5-8), while the wild-type H2A (HDEwt) substrate is processed efficiently in the same extract (lanes 13-16). This finding is in complete agreement with the in vivo data, where little increase in processing at the correct $3^{\prime}$ end of the RNA was observed in the presence of the suppressor U7 (U7uuu $\rightarrow$ aaa) (HDEaaa $\rightarrow$ uuu, Fig. 4A, cf. lanes 30 and 31). The conversion of the H2A HDEuuu $\rightarrow$ aaa precursor to product was $\sim 10 \%$ greater in an extract expressing a noncomplementary U7 suppressor gene (U7py $\rightarrow$ pu) (Fig. 5C, lanes 9-12), but this is because of a difference in the activity of the individual extracts; the U7py $\rightarrow$ pu extract processed the HDEwt substrate with $\sim 10 \%$ greater efficiency than the HDEaaa $\rightarrow$ uuu extract (quantitated from Fig. 5A, lanes 13-16). 
A comparison of the results obtained from the in vitro analyses of the histone mutants and their suppressor U7 RNAs with those obtained in vivo is given in Table 1.

\section{Discussion}

The experiments presented were designed to test the hypothesis that the mammalian U7 snRNP functions in the 3 '-end processing of histone pre-mRNAs through a base-pairing interaction between its $5^{\prime}$ end and the variable mammalian HDE. Our suppression data provide clear confirmation of the existence of base-pairing. Of the 11 nucleotides in the HDE of the H2A-614 transcript (AAAGAGUUGUG) that can potentially pair with mammalian $U 7$, the 6-nucleotide purine stretch appears critical as its deletion or substitution yields no $3^{\prime}$-end processing either in vivo or in vitro. We observe that the more deleterious the effect of a mutation on $3^{\prime}$-end processing, the greater is its ability to be suppressed effectively by a U7 RNA that restores base-pairing with the mutant HDE. Suppression always yields cleavage at the correct site. Most striking is our finding that even the drastic substitution of 6 pyrimidines for the 6 purines of the HDE can be successfully suppressed by compensatory changes in U7 RNA. These findings have important mechanistic implications for the role of the snRNP-substrate interaction in this particular RNA-processing event.

Mutations within the H2A HDE abolish mRNA 3'-end formation in vitro and in vivo

Our finding that a 9-nucleotide deletion, including the 6 purines (AUAAAGAGU) in the HDE of the H2A-614 transcript, effectively abolished 3 '-end processing in both a HeLa cell nuclear extract and in vivo (Figs. 2 and 4) is in agreement with previous observations of a similar mutation in a mouse H3 gene (H3.2-614; Fig. 1) (Mowry et al. 1989). Likewise, substituting pyrimidines (UUUCUC) for the entire purine tract (AAAGAG) or CUC for three of the bases (GAG) yielded no detectable 3 '-end cleavage in vitro; $20 \%$ processing was observed for the latter in vivo (see Table 1). In contrast, substituting UUU for AAA only decreased processing to $20-30 \%$ in vitro and $30-50 \%$ in vivo. Thus, GAG appears to be more important than AAA, with all 6 purines being required for efficient processing.

\section{Expression of synthetic mammalian U7 genes}

A number of interesting findings emerge from our analyses of the synthetic U7 genes constructed with the U1 promoter and downstream sequences required for 3 '-end formation. First, despite numerous differences between the synthetic and endogenous U7 RNAs, stable cell lines both expressed the transfected genes and assembled the resulting RNAs into Sm snRNP particles (Fig. 3). Second, even when expressed from a Ul promoter, the levels of U7syn and its mutants were similar to those of the endogenous U7. U1 RNA is $\sim 1000$ times more abundant than U7 in human cells (Mowry and Steitz 1987b), and there are probably not more than 30 real U1 genes in the human genome (Lund and Dahlberg 1984). Thus, posttranscriptional mechanisms may regulate the abundance of U7 RNA. Third, our RNase mapping of the U7 transcripts produced from the transfected genes revealed not only RNAs of the expected size but also molecules extended at the $3^{\prime}$ end. Only the shorter RNAs are assembled into anti-Sm immunoprecipitable particles, suggesting a link between precise $3^{\prime}$-end formation and the binding of $(\mathrm{Sm})$ proteins to the U7 snRNA. Finally, our U7syn contained several changes in the only potential stem structure in the RNA molecule; these alterations were designed to maintain base-pairing and the same $\Delta \mathrm{G}$ as the normal stem. Previously, Gilmartin et al. (1988) showed that while the secondary structure must be maintained, all bases within the $3^{\prime}$ stem-loop structure of sea urchin U7 could be changed without affecting its biological activity in Xenopus oocytes. In four human U7 pseudogenes, most of the differences with the human U7 sequence derived by Mowry and Steitz (1987b) lie in the region of the stem-loop but maintain dyad symmetry (Soldati and Schümperli 1990). Likewise, a comparison of the human, mouse, and sea urchin $\mathrm{U} 7$ sequences reveals that the region of least conservation is the $3^{\prime}$ stem-loop. Taken together, these results suggest that all U7 RNAs require a 3 '-terminal stem-loop, but the exact sequences within the stem are not important.

\section{Even drastic mutations in the HDE can be suppressed} by compensatory changes in $U 7$

By cotransfection of complementary H2A-614 and U7 mutations, we obtained a number of cell lines in which suppression could be assessed in vivo. The level of suppression was determined by comparing the ratio of correctly processed to readthrough $\mathrm{H} 2 \mathrm{~A}$ transcripts in the presence and absence of a suppressor U7 gene (Fig. 4A). In the case of the HDEpu $\rightarrow$ py mutant, the presence of the U7py $\rightarrow$ pu gene increased the fraction of mRNAs with $3^{\prime}$ ends at the correct site from $<5 \%$ (usually undetectable) to $80-90 \%$ in three cell lines and to $20-50 \%$ in four other lines. Similarly, for the HDEgag $\rightarrow$ cuc mutant, coexpression with the U7cuc $\rightarrow$ gcg suppressor gene reproducibly increased correct processing from $\sim 20 \%-60 \%$. Although it could be argued that the U7 suppressor mutations somehow preferentially destabilize the readthrough transcripts (rather than enhancing processing at the correct site), the ability of the same U7 suppressor RNAs to increase in vitro cleavage of the mutant HDE substrates (Fig. 5) argues against this explanation.

In contrast to the $\mathrm{U} 7 \mathrm{py} \rightarrow \mathrm{pu}$ and $\mathrm{U} 7 \mathrm{cuc} \rightarrow \mathrm{gcg}$ suppressors, the U7uuu $\rightarrow$ aaa mutant did not significantly enhance processing of the corresponding $\mathrm{H} 2 \mathrm{~A}$ HDEaaa $\rightarrow$ uuu mutant in vivo (Fig. 4A), although in some experiments a modest increase was detected. The same result was obtained in in vitro suppression studies (Fig. 5C; Table 1). We believe that this minimal suppression may be explained in terms of the behavior of the 
HDEaaa $\rightarrow$ uuu mutant itself, whose processing both in vivo and in vitro is decreased relative to wild type but not abolished. We infer that the endogenous U7 snRNP can bind to the HDEaaa $\rightarrow$ uuu pre-mRNA to some degree; however, unproductive or inefficient complexes would be formed that would therefore compete and interfere with the ability of the suppressor $\mathrm{U} 7$ to bind, yielding little or no suppression. (In support of this idea, we observe that cell lines expressing higher amounts of the suppressor U7 RNAs give reproducibly higher levels of in vivo suppression.) In contrast to the HDEaaa $\rightarrow$ uuu mutation, the HDEpu $\rightarrow$ py and HDEgag $\rightarrow$ cuc mutations have a much more deleterious effect on 3 ' processing, presumably because wild-type U7 cannot bind efficiently. Consequently, there is little competition from the endogenous U7 snRNP and the appropriate U7 suppressors are capable of effectively enhancing the processing of the mutant HDEpu $\rightarrow$ py and HDEgag $\rightarrow$ cuc substrates both in vivo and in vitro. The fact that HDEgag $\rightarrow$ cuc is less suppressible than HDEpu $\rightarrow$ py may be because of partial competition from the endogenous U7, which could base-pair with the unchanged AAA. Alternatively, a stretch of 6 purines or pyrimidines as exemplified by the HDEpu $\rightarrow$ py mutant may be more favorable to processing than mixed purines and pyrimidines as found in the HDEgag $\rightarrow$ cuc mutant.

In addition to reproducing the in vivo suppression results, the in vitro system allowed us to test the specificity of suppression (Fig. 5). We found that a particular H2A mutant cannot be suppressed by any suppressor; only a U7 with the correct compensatory changes is active. Exceptions to this generalization are the suppression of the $\mathrm{H} 2 \mathrm{~A}$ HDEgag $\rightarrow$ cuc mutant by the U7cuc $\rightarrow$ gcg suppressor (Figs. $4 A$ and $5 B$ ) and to a lesser extent by the U7py $\rightarrow$ pu suppressor (not shown). Suppression with a single mismatch within the potential base-paired region (Fig. 4B) is in accord with the variability of HDEs of mammalian histone genes (Fig. 1). Suppression by $\mathrm{U} 7 \mathrm{py} \rightarrow$ pu can be explained by restoration of base-pairing between the CUC in the HDE with the GAG in the U7py $\rightarrow$ pu while the AAA in the HDE remains unpaired; we argued above that pairing of the AAA is not critical for processing. It is not clear why the efficiency of suppression obtained in vitro is so much lower than that achieved in vivo, although similar differences have been observed in yeast pre-mRNA splicing (McPheeters et al. 1989). Perhaps in vitro suppression could be enhanced by inactivating the endogenous $U 7$ snRNPs with specific cleavage with RNase $\mathrm{H}$.

\section{Mechanistic implications for U7 snRNP function}

The two interactions investigated most thoroughly between snRNAs and specific sequences in pre-mRNAs are those of U1 snRNA with the $5^{\prime}$-splice site and U2 snRNA with the intron branchpoint during pre-mRNA splicing. In the mammalian system, Zhuang and Weiner (1986) showed that mutations in some but not all positions in the 5 '-splice site sequence can be suppressed by a compensatory mutation at the corresponding position of U1 RNA. Similar mutational analyses of the $5^{\prime}$-splice sites of yeast pre-mRNAs revealed that while base-pairing with $\mathrm{Ul}$ is necessary, it is not sufficient for efficient and correct splicing (Séraphin et al. 1988; Siliciano and Guthrie 1988; Séraphin and Rosbash 1989, 1990). Yet, the efficiency of cleavage at the $5^{\prime}$-splice site was increased in yeast cells containing the appropriate $U 1$ compensatory mutations. Studies testing base-pairing between the intron branchpoint and U2 snRNA have likewise concluded that while base-pairing is important, complementarity alone is not sufficient for efficient and accurate splicing in either yeast or human cells (Parker et al. 1987; McPheeters et al. 1989; Wu and Manley 1989; Zhuang and Weiner 1989). It appears that either the precise nature of the base-pairing interactions involving the $U 1$ and $U 2$ snRNPs or the recognition of these pre-mRNA regions by other splicing factors is critical for spliceosome function.

Our manipulation of the base-pairing interaction between mammalian U7 snRNA and the HDE of the H2A histone pre-mRNA has yielded results that are quite different from those summarized above. Even the drastic substitution of 6 pyrimidines for 6 adjacent purines within the HDE is detectably suppressed by compensatory changes in U7. Presumably this is because the basepaired sequences do not overlap the site of cleavage in the histone pre-mRNA, whereas in the splicing reaction the first step of catalysis involves nucleotides that reside within the regions of base-pairing with $\mathrm{U} 1$ and $\mathrm{U} 2$ snRNAs. Yet, suppression of the HDE mutations was not complete, either in vivo or in vitro. If the binding of the U7 snRNP serves only to orient other processing components for cleavage, then any base-pairing, as long as it is of sufficient stability, should suffice for efficient histone mRNA 3 '-end maturation. The fact that this is not the case argues that other components of the system may be sensitive to the nature of the base-paired nucleotides. Accordingly, our observation that the GAG substitution is much more deleterious than the AAA substitution could be due to the stronger base pairs formed by GAG, to its more central position in the HDE of the H2A-614 pre-mRNA, or to the interaction of protein(s) with these particular base pairs in the processing complex. Because we have not yet replaced every position of the HDE with all three other bases and tested suppression, we cannot distinguish the relative importance of these contributions. It is also possible that we would have obtained even better suppression had we retained the wild-type 3 '-stem sequence in the suppressor U7 RNAs.

Our studies confirm that the position of the base-pairing interaction with $U 7$ relative to the cleavage site is important as our HDE $\Delta 9$ mutant, in which 9 nucleotides of the HDE are deleted, retains as much base-pairing potential as some histone pre-mRNAs /cf. Fig. 2B and Fig. 1) but is not processed either in vivo or in vitro. It has been shown previously that a 6 -base insertion between the 3 '-cleavage site and the HDE of a sea urchin $\mathrm{H} 3$ gene abolishes all processing (Georgiev and Birnstiel 1985). Further studies are required to define precisely the ex- 
tent of flexibility allowed in the position of the basepairing interaction with the mammalian U7 snRNP.

The absence of a highly defined sequence requirement for the U7 snRNP-pre-mRNA base-pairing interaction is compatible with the diversity of HDE sequences found in mammalian histone genes. An alternative possibility, that multiple U7 RNAs with different 5 '-end sequences exist, appears unlikely on the basis of our results. The fact that all HDE elements contain a purine stretch may be due to evolutionary inertia, but the type of base-pairing appears to influence the efficiency of the reaction. It remains to be determined what the minimal base-pairing between the mammalian HDE and U7 is for processing to occur and whether the regulation of 3 '-end formation is sensitive to the nature of the interaction.

\section{Materials and methods}

\section{Construction of H2A HDE mutants}

The mouse H2A-614 gene (Hurt et al. 1989), contained within a 1650-bp EcoRI-XbaI fragment, was kindly provided by W. Marzluff (Florida State University) [Fig. 6, H2A-614 (HDEwt)]. The promoter sequences (nucleotides 1-813) are represented by a black line; the mRNA-coding sequences (nucleotides 814-1328) are denoted by the shaded area. The plasmid also contains 322 nucleotides downstream of the $3^{\prime}$ end of the RNA (hatched area). To generate mutations in the HDE, a SacI-XbaI fragment (351 nucleotides) was replaced by a shorter synthetic fragment (111 nucleotides), which was flanked by $S a c I$ and $X b a I$ restriction sites at the $5^{\prime}$ and $3^{\prime}$ ends, respectively, and contained the mutated bases within the HDE (see Fig. 2A). The SacI site (at nucleotide 1299 relative to the EcoRI site at nucleotide 1 ) is 29 nucleotides upstream of the $3^{\prime}$ end of the mRNA. The mutant genes (H2A HDE mutants; Fig. 6) therefore consist of bases 1-1410 of the H2A-614 (HDEwt) gene with the mutations in the HDE incorporated. The resultant clones were sequenced by using the dideoxy sequencing procedure (Sanger et al. 1977).

To produce antisense RNAs as protection probes for the HDEwt and HDE $\Delta 9$ transcripts, a PvuI-XbaI fragment $484 \mathrm{nu}$ cleotides for HDEwt and 240 nucleotides for HDE $\Delta$ 9; Fig. 6) was subcloned into a Bluescript SK vector (Stratagene) at the SmaI and $\mathrm{XbaI}$ sites in the polylinker. To ligate the fragment at the SmaI site, the PvuI site was first blunt-ended with T4 DNA polymerase. The HDEaaa $\rightarrow$ uuu RNase protection probe was obtained by subcloning the BalI-XbaI fragment $(295$ nucleotides; see Fig. 6) into a Bluescript SK plasmid at the HincII and
$X b a I$ sites in the polylinker. The HDEpu $\rightarrow$ py Ball-XbaI fragment was subcloned into Bluescript SK at the NotI and XbaI sites in the polylinker. The NotI site was first filled in with Klenow fragment (see Fig. 8, below). The plasmid constructs were verified by dideoxy sequencing.

To generate transcripts for in vitro processing, the SacI-XbaI fragment of the HDEwt (354 nucleotides) and the HDE mutants (111 nucleotides) was cloned into a Bluescript SK vector at the $S a c I$ and $X b a I$ sites in the polylinker.

\section{Construction of U7syn and U7 suppressor mutants}

The U7syn gene was generated by preparing synthetic oligonucleotides containing the required sequences. The sense strand consists of 12 random anchor residues $\left(N_{12}\right)$ at the $5^{\prime}$ end, residues -7 to +2 of the human $U 1$ gene (which happen to contain a BgIII restriction site), nucleotides 3-63 of the human U7 RNA sequence (Mowry and Steitz 1987b), nucleotides 1-27 downstream of the human U1 gene, a SalI restriction site, and 11 additional random anchor $\left(\mathrm{N}_{11}\right)$ residues. The sequence of the sense-strand oligonucleotide is shown in Figure 7A. The U1derived nucleotides are marked, and the restriction sites used for cloning are shown. The predicted $U 7$ transcript is boxed; +1 marks the first base in the transcript and +63 marks the last base. The bases in the U7 that differ from the human U7 sequence are underlined. The oligonucleotides were annealed, phosphorylated at their 5' ends, and digested with BglII and Sall. The plasmid pUC/Ul (Fig. 7B), kindly provided by R. Ach (Yale Universityl, contains 425 nucleotides of upstream sequences (hatched area), the U1 sequence (area shaded black), and $90 \mathrm{nu}-$ cleotides of downstream sequences (hatched area). Digestion with $B g l I I$ and SalI removed the Ul gene and the downstream sequences. The BgIII-Sall fragment was then replaced with the annealed, BglII- and SalI-digested, synthetic oligonucleotides containing the U7 sequence (lightly hatched area) to yield U7syn in pUC (Fig. 7B).

To generate the suppressor mutations, the BamHI-Sall fragment of U7syn was cloned into M13mp18, and mutagenesis was performed as described by Kunkel (1985). The mutations were identified by dideoxy sequencing. The BamHI-Sall fragments of the mutated genes were then cloned into Bluescript SK plasmids by using the BamHI and SalI sites. The synthetic U7 (U7syn) gene in pUC and the mutated U7 genes in Bluescript SK were used for transfection into HeLa cells.

\section{Transfection of HeLa cells}

The H2A- and U7-containing plasmids either singly (10 $\mu \mathrm{g})$ or together $(5 \mu \mathrm{g}$ each), along with the neomycin gene $(1 \mu \mathrm{g})$ [pSVneo (Southern and Berg 1982), kindly provided by Dr. S.
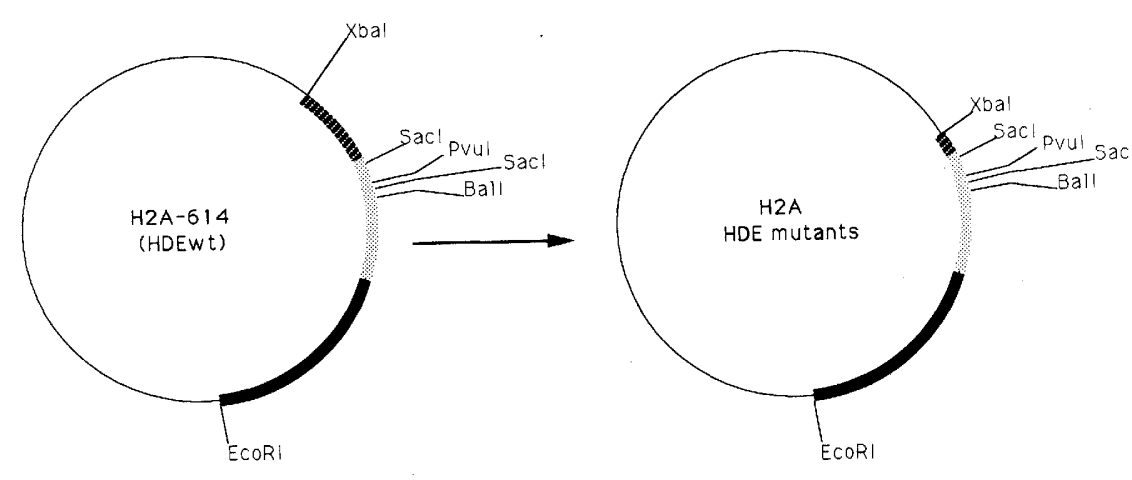

Figure 6. Plasmid map of H2A-614 (HDEwt) and the H2A HDE mutants. 


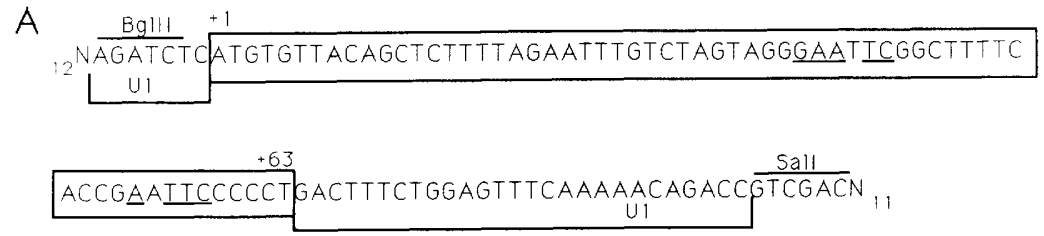

B

Figure 7. (A) Sequence of the sense strand oligonucleotide used to generate the U7syn gene; $(B)$ plasmid map of pUC/U1 and the derivative clone U7syn.

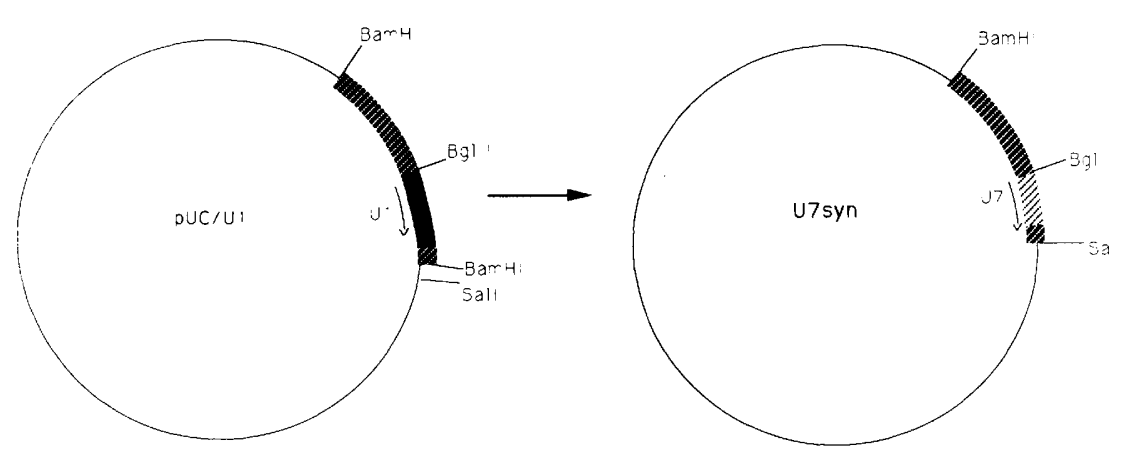

Baserga, Yale Universityl, were incubated with lipofectin reagent and transfected into HeLa cells as described by the manufacturers (Bethesda Research Laboratories). Colonies resistant to $\mathrm{G} 418(1 \mathrm{mg} / \mathrm{ml})$ were selected. To obtain a cell line coexpressing the H2A HDEpu $\rightarrow$ py and the U7py $\rightarrow$ pu genes, a cell line that already contained the H2A HDEpu $\rightarrow$ py gene and that was G-418 resistant was subsequently transfected with the $\mathrm{U} 7 \mathrm{py} \rightarrow \mathrm{pu}$ gene and a hygromycin B phosphotransferase gene [Blochlinger and Diggelmann (1984), kindly provided by Dr. J. Sedivy, Yale University] as described above. Clones of cells resistant to hygromycin $(150 \mu \mathrm{g} / \mathrm{ml})$ were selected and propagated.
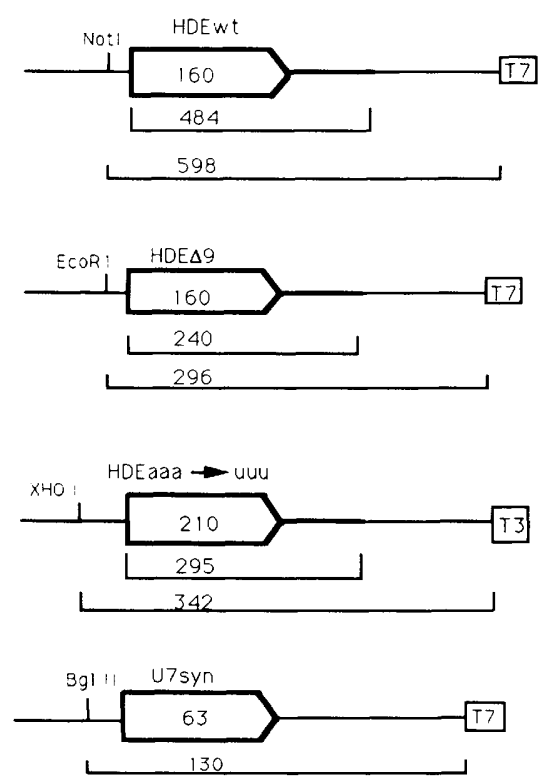

Figure 8. Probes used for RNase $\mathrm{T} 1$ protection assays.

\section{RNase protection}

Antisense RNA probes for the HDEwt, the HDE mutants, and the U7 sequences were prepared by $\mathrm{T} 3$ or T7 RNA polymerase runoff transcription (Yisraeli and Melton 1989) from the plasmid constructs described above. The restriction sites used to generate the runoff transcripts and the sizes of the transcripts (second line below diagram) are shown in Figure 8. The expected sizes for protection of correctly processed RNA are shown within the box and the length of the H2A sequences within the transcripts (represented as boldface lines) are indicated in the first line below the diagram. The U7 antisense probes are all identical except for the mutated sequences in the polypyrimidine stretch. RNase protection assays were performed as described by Howe and Shu (1989) except that $50 \mu \mathrm{g}$ of RNA, $1 \times 10^{6} \mathrm{cpm}$ of probe, and 34 units of RNase T1 were used per reaction. Quantitation of the protected fragments was performed by using a Phosphorimager (Molecular Dynamics).

\section{In vitro processing}

RNA transcripts for HDEwt, HDEpu $\rightarrow$ py, HDEgag $\rightarrow$ cuc, and HDEaaa $\rightarrow$ uuu were generated by T3 RNA polymerase runoff transcription of the SacI-XbaI subclones /see Construction of H2A HDE mutants, above) at the MaeIII site (which is $24 \mathrm{nu}-$ cleotides downstream of the processing site) and at the HpaII site (which is 36 nucleotides downstream of the processing site) for $\operatorname{HDE} \Delta 9$. The transcripts contain 14 nucleotides of vector sequences upstream of the $\mathrm{H} 2 \mathrm{~A}$ sequences. The sizes of the runoff transcripts were 69 nucleotides for HDEwt, HDEpu $\rightarrow$ py, HDEgag $\rightarrow$ cuc, and HDEaaa $\rightarrow$ uuu and $72 \mathrm{nu}-$ cleotides for HDE $\Delta 9$. Nuclear extracts were prepared as described by Heintz and Roeder (1984), except that cells were frozen in liquid nitrogen after washing in phosphate-buffered saline. The processing reactions were carried out as described previously (Mowry and Steitz 1987a) and contained $10^{-16}$ to $10^{-15}$ moles of transcript per $6-\mu \mathrm{l}$ extract. In experiments where the amount of extract was varied, the $\mathrm{KCl}$ concentration was adjusted to give a final concentration of $80 \mathrm{~mm}$. 


\section{Anti-Sm precipitation of snRNAs}

Nuclear extracts were prepared from cell lines as described above. Twelve microliters $\left(\sim 4 \times 10^{6}\right.$ cell equivalents) was incubated with $24 \mu$ lof Y-12 monoclonal antibody (anti-Sm) that was prebound to protein A-Sepharose (Pharmacia). Immunoprecipitation was performed and RNA was extracted from the pellets and supernatants as described by Mowry and Steitz (1987a). Half of the extracted RNA $\left(2 \times 10^{6}\right.$ cell equivalents) was then used for RNase protection with antisense $U 7$ probes as described by Howe and Shu (1989).

\section{Acknowledgments}

This work was supported in part by a grant to J.A. S. from the National Institutes of Health (GM26154), and initial stages of this work were supported by an Anna Fuller postdoctoral fellowship to U.M.B. We thank W. Marzluff for his kind gift of the H2A-614 plasmid DNA and for the compilation of the mouse 3 '-end sequences. We are grateful to Alan Weiner, Jacqueline Wyatt, David Wassarman, Karen Wassarman, and T.C. James for helpful suggestions on this manuscript and to Lynda Stevens for typing the manuscript.

The publication costs of this article were defrayed in part by payment of page charges. This article must therefore be hereby marked "advertisement" in accordance with 18 USC section 1734 solely to indicate this fact.

\section{References}

Birnstiel, M.L. and F.J. Schaufele. 1988. Structure and function of minor snRNPs. In Structure and function of major and minor small nuclear ribonucleoprotein particles. (ed. $\mathbf{M}$. Birnstiel), pp. 155-162, Springer-Verlag, New York.

Blochlinger, K. and H. Diggelmann. 1984. Hygromycin B phosphotransferase as a selectable marker for DNA transfer experiments with higher eukaryotic cells. Mol. Cell. Biol. 4: 2929-2931.

Cotten, M., O. Gick, A. Vasserot, G. Schaffner, and M.L. Birnstiel. 1988. Specific contacts between mammalian U7 snRNA and histone precursor RNA are indispensable for the in vitro 3' RNA processing reaction. EMBO 1. 7: 801-808.

Galli, G., H. Hofstetter, H.G. Stunnenberg, and M.L. Birnstiel. 1983. Biochemical complementation of RNA in the Xenopus oocyte: A small RNA is required for the generation of $3^{\prime}$ histone mRNA termini. Cell 34: 823-828.

Georgiev, O. and M.L. Birnstiel. 1985. The conserved CAAGAAAGA spacer sequence is an essential element for the formation of $3^{\prime}$ termini of the sea urchin $\mathrm{H} 3$ histone mRNA by RNA processing. EMBO $T$. 4: 481-489.

Gick, O., A. Krämer, A. Vasserot, and M.L. Birnstiel. 1987. Heat-labile regulatory factor is required for $3^{\prime}$ processing of histone precursor mRNAs. Proc. Natl. Acad. Sci. 84: 89378940.

Gilmartin, G.M., F. Schaufele, G. Schaffner, and M.L. Birnstiel. 1988. Functional analysis of the sea urchin U7 small nuclear RNA. Mol. Cell. Biol. 8: 1076-1084.

Graves, R.A., S.E. Wellman, I.M. Chiu, and W.F. Marzluff. 1985. Differential expression of two clusters of mouse histone genes. J. Mol. Biol. 183: 179-194.

Gruber, A., A. Streit, M. Reist, P. Benninger, R. Böhni, and D. Schümperli. 1990. Structure of a mouse histone-encoding gene cluster. Gene 95: 303-304.

Heintz, N. and R.G. Roeder. 1984. Transcription of human histone genes in extracts from synchronized HeLa cells. Proc.
Natl. Acad. Sci. 81: 2713-2717.

Heintz, N., H.L. Sive, and R.G. Roeder. 1983. Regulation of human histone gene expression: Kinetics of accumulation and changes in the rate of synthesis and in the half-lives of individual histone mRNAs during the HeLa cell cycle. Mol. Cell. Biol. 3: 539-550.

Hernandez, N. 1985. Formation of the $3^{\prime}$-end of U1 RNA is directed by a conserved sequence located downstream of the coding region. EMBO I. 4: 1827-1837.

Howe, J.G. and M.-D. Shu. 1989. Epstein-Barr virus small RNA (EBER) genes: Unique transcription units that combine RNA polymerase II and III promoter elements. Cell 57: 825-834.

Hurt, M.M., N. Chodchoy, and W.F. Marzluff. 1989. The mouse histone H2a.2 gene from chromosome 3. Nucleic Acids Res. 17: 8876 .

Kunkel, T. 1985. Rapid and efficient site specific mutagenesis without phenotypic selection. Proc. Natl. Acad. Sci. 82: 488-492.

Levine, B.J., T.-J. Liu, W.F. Marzluff, and A.I. Skoultchi. 1988. Differential expression of individual members of the histone multigene family due to sequences in the $5^{\prime}$ and $3^{\prime}$ regions of the genes. Mol. Cell. Biol. 8: 1887-1895.

Liu, T.-I., L. Liu, and W.F. Marzluff. 1987. Mouse histone H2A and $\mathrm{H} 2 \mathrm{~B}$ genes: Four functional genes and a pseudogene undergoing gene conversion with a closely linked functional gene. Nucleic Acids Res. 15: 3023-3039.

Liu, T.-J., B.J. Levine, A.I. Skoultchi, and W.F. Marzluff. 1989. The efficiency of 3 '-end formation contributes to the relative levels of different histone mRNAs. Mol. Cell. Biol. 9: 34993508.

Lund, E. and J. Dahlberg. 1984. True genes for human U1 small nuclear RNA. J. Biol. Chem. 259: 2013-2021.

Lüscher, B. and D. Schümperli. 1987. RNA 3' processing regulates histone mRNA levels in a mammalian cell cycle mutant. A processing factor becomes limiting in Gl-arrested cells. $E M B O$ /. 6: 1721-1726.

Lüscher, B., C. Stauber, R. Schindler, and D. Schümperli. 1985. Faithful cell-cycle regulation of a recombinant mouse histone $\mathrm{H} 4$ gene is controlled by sequences in the $3^{\prime}$-terminal part of the gene. Proc. Natl. Acad. Sci. 82: 4389-4393.

Mannironi, C., W.H. Bonner, and C.L. Hatch. 1989. H2A.X. a histone isoprotein with a conserved C-terminal sequence, is encoded by a novel mRNA with both DNA replication type and polyA $3^{\prime}$ processing signals. Nucleic Acids Res. 17: 9113-9126.

Manser, T. and R.F. Gesteland. 1981. Characterization of small nuclear Ul gene candidates and pseudogenes from the human genome. J. Mol. Appl. Genet. 1: 117-125.

Marzluff, W.F. and N.B. Pandey. 1988. Multiple regulatory steps control histone mRNA concentrations. Trends Biochem. Sci. 13: 49-52.

McPheeters, D.S., P. Fabrizio, and J. Abelson. 1989. In vitro reconstitution of functional yeast U2 snRNPs. Genes \& Dev. 3: 2124-2136.

Meier, V.S., R. Böhni, and D. Schümperli. 1989. Nucleotide sequence of two mouse histone $\mathrm{H} 4$ genes. Nucleic Acids Res. 17: 795.

Mowry, K.L. and J.A. Steitz. 1987a. Both conserved signals on mammalian histone pre-mRNAs associate with small nuclear ribonucleoproteins during $3^{\prime}$-end formation in vitro. Mol. Cell. Biol. 7: 1663-1672.

1987b. Identification of the human U7 snRNP as one of several factors involved in the 3 '-end maturation of histone premessenger RNA's. Science 238: 1682-1687.

- 1988. snRNP mediators of 3 '-end processing: Functional fossils? Trends Biochem. Sci 13: 447-451. 
Mowry, K.L., R. Oh, and J.A. Steitz. 1989. Each of the conserved sequence elements flanking the cleavage site of mammalian histone pre-mRNAs has a distinct role in the $3^{\prime}$-end processing reaction. Mol. Cell. Biol. 9: 3105-3108.

Parker, R., P.G. Siliciano, and C. Guthrie. 1987. Recognition of the TACTAAC box during mRNA splicing in yeast involves base pairing to the U2-like snRNA. Cell 49: 229-239.

Sanger, F., S. Nicklen, and A.R. Coulson. 1977. DNA sequencing with chain terminating inhibitors. Proc. Natl. Acad. Sci. 74: 5463-5476.

Schaufele, F., G.M. Gilmartin, W. Bannwarth, and M.L. Birnstiel. 1986. Compensatory mutations suggest that base-pairing with a small nuclear RNA is required to form the $3^{\prime}$-end of H3 messenger RNA. Nature 323: 777-781.

Seiler-Tuyns, A. and M.L. Birnstiel. 1981. Structure and expression in L-cells of a cloned $\mathrm{H} 4$ histone gene of the mouse. I. Mol. Biol. 151: 607-625.

Séraphin, B. and M. Rosbash. 1989. Mutational analysis of the interactions between Ul small nuclear RNA and pre-mRNA of yeast. Gene 82: 145-151.

1990. Exon mutations uncouple 5'-splice site selection from Ul snRNA pairing. Cell 63: 619-629.

Séraphin, B., L. Kretzner, and M. Rosbash. 1988. A Ul snRNA : pre-mRNA base pairing interaction is required early in yeast spliceosome assembly but does not uniquely define the 5' cleavage site. EMBO I. 7: 2533-2538.

Sierra, F., G. Stein, and J. Stein. 1983. Structure and in vitro transcription of a human $\mathrm{H} 4$ histone gene. Nucleic Acids Res. 11: 7069-7086.

Siliciano, P.G. and C. Guthrie. 1988. 5'-Splice site selection in yeast: Genetic alterations in base-pairing with Ul reveal additional requirements. Genes \& Dev. 2: 1258-1267.

Sittman, D.B., R.A. Graves, and W.F. Marzluff. 1983. Structure of a cluster of mouse histone genes. Nucleic Acids Res. 11: $6679-6697$.

Soldati, D. and D. Schümperli. 1988. Structural and functional characterization of mouse U7 small nuclear RNA active in 3' processing of histone pre-mRNA. Mol. Cell. Biol. 8: 15181524.

- 1990. Structures of four human pseudogenes for U7 small nuclear RNA. Gene 95(2): 305-306.

Southern, P.J. and P. Berg. 1982. Transformation of mammalian cells to antibiotic resistance with a bacterial gene under control of the SV40 early region promoter. I. Mol. Appl. Genet. 1: 327-341.

Stein, G.S., F. Sierra, J.L. Stein, M. Plumb, F. Marashi, N. Carrozzi, K. Prokopp, and L. Baumbach. 1984. Organization and expression of human histone genes. In Histone genes: Structure, organization and regulation (ed. G.S. Stein, J.L. Stein, and W.F. Marzluff), pp. 397-455, John Wiley and Sons, New York.

Strub, K. and M.L. Birnstiel. 1986. Genetic complementation in the Xenopus oocyte: Coexpression of sea urchin histone and U7 RNAs restores $3^{\prime}$ processing of $\mathrm{H} 3$ pre-mRNA in the oocyte. EMBO I. 5: 1675-1682.

Taylor, J.D., S.E. Wellman, and W.F. Marzluff. 1986. Sequences of four mouse histone $\mathrm{H} 3$ genes: Implications for evolution of mouse histone genes. J. Mol. Evol. 23: 242-249.

Vasserot, A.P., F.J. Schaufele, and M.L. Birnstiel. 1989. Conserved terminal hairpin sequences of histone mRNA precursors are not involved in duplex formation with the U7 RNA but act as a target site for a distinct processing factor. Proc. Natl. Acad. Sci. 86: 4345-4349.

Wu, J. and J.L. Manley. 1989. Mammalian pre-mRNA branch site selection by $\mathrm{U} 2$ snRNP involves base-pairing. Genes \& Dev. 3: 1553-1561.
Yang, Y.-S., D.T. Brown, S.E. Wellman, and D.B. Sittman. 1987. Isolation and characterization of a mouse fully replicationdependent $\mathrm{Hl}$ gene within a genomic cluster of core histone genes. J. Biol. Chem. 262: 17118-17125.

Yisraeli, J.K. and D.A. Melton. 1989. Synthesis of long, capped transcripts in vitro by SP6 and T7 RNA polymerase. Methods Enzymol. 180: 42-50.

Zhong, R., R.G. Roeder, and N. Heintz. 1983. The primary structure and expression of four cloned human histone genes. Nucleic Acids Res. 11: 7409-7425.

Zhuang, Y. and A.M. Weiner. 1986. A compensatory base change in U1 snRNA suppresses a $5^{\prime}$-splice site mutation. Cell 46: 827-835.

1989. A compensatory base change in human U2 snRNA can suppress a branch site mutation. Genes \& Dev. 3: $1545-1552$. 


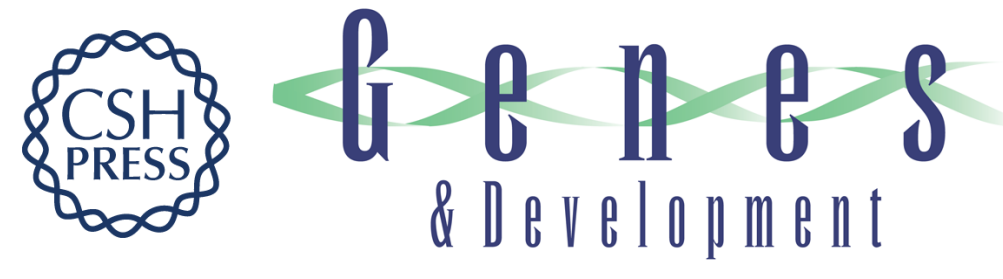

\section{Multiple processing-defective mutations in a mammalian histone pre-mRNA are suppressed by compensatory changes in U7 RNA both in vivo and in vitro.}

U M Bond, T A Yario and J A Steitz

Genes Dev. 1991, 5:

Access the most recent version at doi:10.1101/gad.5.9.1709

References This article cites 51 articles, 21 of which can be accessed free at:

http://genesdev.cshlp.org/content/5/9/1709.full.html\#ref-list-1

License

Email Alerting Receive free email alerts when new articles cite this article - sign up in the box at the top

Service right corner of the article or click here.

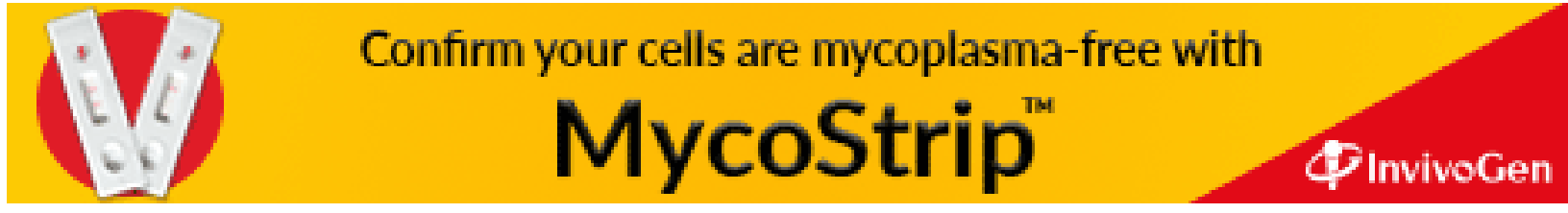

